\title{
Realidades psico-sociales de las mujeres privadas de la libertad en el centro de rehabilitación social femenino Portoviejo "Tomás Larrea"
}

\section{Psycho-social realities of women deprived of liberty in the social rehabilitation center Portoviejo "Tomás Larrea" \\ Realidades psico-sociales de las mujeres privadas de la libertad en el centro de rehabilitación social femenino Portoviejo "Tomás Larrea"}

\author{
Cecilia Mera-Zambrano ${ }^{\mathrm{I}}$ \\ anitacecilia16@hotmail.es \\ Yuliana D. Roca-Castro II \\ yulianadroca@gmail.com
}

Recibido: 30 de enero de 2017 * Corregido: 20 de febrero de 2017 * Aceptado: 20 junio de 2017

\footnotetext{
${ }^{\text {I } D o c e n t e s ~ d e ~ l a ~ F a c u l t a d ~ d e ~ T r a b a j o ~ S o c i a l, ~ U n i v e r s i d a d ~ L a i c a ~ E l o y ~ A l f a r o ~ d e ~ M a n a b i ́, ~ M a n t a, ~ E c u a d o r . ~}$

${ }^{\text {II }}$ Docentes de la Facultad de Trabajo Social, Universidad Laica Eloy Alfaro de Manabí, Manta, Ecuador.
} 


\section{Resumen}

En esta investigación se ha abordado el tema titulado: Condiciones psico-sociales de las mujeres privadas de la libertad en el Centro de Rehabilitación Social Femenino "Tomas Larrea”, la misma que identifica las condiciones psico-sociales de las personas recluidas en este lugar. El problema se centra en la forma de como la prisión afecta las diferentes condiciones humanas al momento de cumplir la condena que le impone la ley y la sociedad.

Las condiciones sociales y psicológicas manifestadas por la privación de la libertad se muestran en la convivencia carcelaria donde aluden pérdidas profundas, dolorosas rupturas y mortificaciones extremas, reforzándose diariamente en la mujer, una nueva identidad en un mundo diferente, que se construye opuestamente al hecho de estar fuera y de ser libres. La hipótesis planteada determina que la privación de libertad de las mujeres del Centro de Rehabilitación Social Femenino Portoviejo "Tomas Larrea", influye de forma negativa a las condiciones psico sociales. La muestra de trabajo fue de 33 internas. La investigación es realizada con conocimiento científico, descriptivo, método inductivo, deductivo, analítico; para la toma de datos se utilizó la observación, entrevistas, encuestas y estudios de casos.

Como conclusión se obtuvo, que el encierro produce, trastornos de conductas y personalidad, como depresión, soledad, desvalorización personal, violencias, intentos suicidas, vulnerabilidad al consumo de sustancias psicotrópicas, rechazo social, alejamiento familiar, desvinculación laboral y económica.

Palabras clave: Mujeres privadas de libertad, condiciones sociales y psicológicas, prisión, reclusión, privación de libertad. 


\section{Abstract}

In this research paper entitled: psycho social conditions of women deprived of liberty in the Social Rehabilitation Center Women Portoviejo "Tomas Larrea," identifies the psychosocial conditions of women deprived of liberty; the problem focuses on describing as the closure affects the different conditions of the woman prisoner.

The social and psychological conditions expressed by the deprivation of liberty, shown in coexistence prison where allude deep, painful losses, breaks and extreme mortification, reinforced daily in women, a new identity in a different world, which is built on opposition to being out and be free. The hypothesis determines that the imprisonment of women Social Rehabilitation Center Women Portoviejo "Tomas Larrea" adversely affects the Psycho Social conditions. The universe of study was 33 of 43 inmates. The research is conducted with scientific, descriptive, inductive, deductive, analytical method knowledge; for data collection, observation, interviews, surveys, and case studies are used.

In conclusion was obtained that the closure occurs, behavioral and personality disorders, such as depression, loneliness, personal devaluation, violence, suicide attempts, vulnerability to substance use, misuse of sexual roles, social rejection, employment and economic decoupling.

Key words: Women, prisoners, social and psychological conditions, imprisonment, confinement, deprivation of liberty. 


\section{Introducción.}

La privación de libertad es un acto que se da a las personas, que han transgredido algún hecho perjudicial a la sociedad, después de esta percepción, la cárcel, constituye para las mujeres un espacio discriminativo y opresivo, que se manifiesta en la desigualdad de trato que reciben en relación a los varones, el encierro y las consecuencias, la forma como la administración de justicia opera frente a sus casos, en las concepciones que la sociedad en general atribuye a las mujeres que se encuentran en estas circunstancias. (Estibaliz de Miguel, 2014)

El hombre interactúa en un medio que incide en su comportamiento, estableciéndolo como un ser social. Así se produce un proceso dialéctico inevitable, para el desarrollo continuo de la sociedad e influye en las acciones que realiza de una u otra manera con las relaciones que mantiene en diferentes ámbitos de la vida cotidiana. (Boldo, 2014)

El presente estudio tiene como objetivo general investigar las condiciones psico sociales de las mujeres que permanecen recluidas en el Centro de Rehabilitación Social Femenino Portoviejo “Tomas Larrea", enfocado en la experiencia de las mujeres privadas de libertad, que se dan dentro del reclusorio, se plantean objetivos específicos como determinar los imaginarios que circulan sobre los estigmas de la mujer común, "mujer, esposa, madre", generando en ellas cambios físicos, psicológicos y sociales, realizar un análisis de cómo el aislamiento afecta a las mujeres privadas de libertad y cómo ellas afrontan esta situación.

Cada mujer es una síntesis del mundo patriarcal: de sus normas, de sus prohibiciones, de sus deberes, de los mecanismos pedagógicos (sociales, ideológicos, afectivos, intelectuales, políticos) 
que internan en ella el ser mujer y enfrenta diversas situaciones que surgen en el desarrollo de la vida diaria dentro del entorno donde se desenvuelven.

La privación de libertad es una condena perversa, para los hombres y las mujeres, ambos son víctimas del encierro, de los prototipos que la sociedad impone; sin embargo, no se puede desconocer que las mujeres encarceladas son víctimas de una triple discriminación: la que se deriva por su condición de féminas; la que les impone su situación de encierro y la que es común a todos los grupos de personas vulnerables y excluidas. (Mapelli, Borja Herrera, \& Sordi Stock, 2013)

Se observa que la población mundial de mujeres privadas de libertad crece diariamente convirtiéndose en una preocupación social y política para los gobiernos. Por ende, se debe atribuir importancia a las mujeres que están privadas de la libertad, al estar recluidas son separadas de la familia provocando cambios drásticos en la vida de cada una de ellas y en el vínculo familiar.

En lo que corresponde a delincuencia, las mujeres presentan índices más bajos que los hombres, no suele superar el 10\% con relación al total de la población privada de libertad en todos los países, reflejando que influyen otras causas asociadas a la condena que deben cumplir por diferentes aspectos (Rodriguez, 2015).

En Ecuador las cárceles no brindan las condiciones de vida adecuadas a las mujeres privadas de la libertad, existe hacinamiento, inseguridad, desigualdad de género y sobre todo no se ejecutan programas de rehabilitación social que les permita reinsertarse a su entorno una vez que cumplan su condena.

Según la Constitución de la República del Ecuador 2008, el Estado ejercerá la rectoría del sistema a través de la Autoridad Sanitaria Nacional, será responsable de formular la política nacional 
de salud, normará, regulará y controlará todas las actividades relacionadas a la salud, así como el funcionamiento de las entidades del sector, haciendo referencia que en el país la competencia de salud penitenciaria se llevaba a cabo de manera parcial por la cartera de justicia.

El Estado Ecuatoriano garantiza lo expuesto en el Artículo 35, Derechos de las personas y grupos de atención prioritaria en donde se señala "Las personas adultas mayores, niñas, niños y adolescentes, mujeres embarazadas, personas con discapacidad, personas privadas de libertad y quienes adolezcan de enfermedades catastróficas o de alta complejidad, recibirán atención prioritaria y especializada en los ámbitos público y privado" (Constitución de la República del Ecuador, 2008).

Entre los lineamientos estratégicos y las políticas del Objetivo 6, del Plan Nacional del Buen Vivir (2013-2017), se encuentra la de Consolidar la transformación del sistema de rehabilitación social, en cuyo literal "e" hace referencia a Promover acciones de reinserción social para las personas puestas en libertad, donde la intervención psicológica, en sentido amplio, se presenta como elemento nuclear e indispensable.

Las causas que pueden ser identificadas incide en que el investigador determine los riegos psico-sociales que se presentan en la muestra escogida, su relación con trastornos mentales u otros que pueden surgir por el encierro en que se encuentran, su comportamiento influye dependiendo del entorno social donde ha desarrollado las etapas de su vida, tomando en cuenta que el factor familia es el más importante para hacer frente a la situación presentada, mujeres que han cometido sus actos porque han sufrido graves consecuencias como violencia de pareja, falta de afecto y apoyo de padres, bajos recursos económicos que las inducen a realizar acciones no adecuadas. 
Considerando lo antes mencionado surge la importancia de investigar los aspectos más relevantes que influyen en la vida diaria de las mujeres privadas de la libertad y cómo se desenvuelven en ese entorno en que viven, tomando en cuenta que el Estado en los últimos años ha mejorado las condiciones de alimentación y salud, garantizando los derechos establecidos en la Constitución de la República.

Nuestro país no cuenta con estudios que permitan analizar las características de las condiciones penitenciarias donde se pueda conocer la vulneración de derechos que existe en las mujeres privadas de la libertad que las diferencian de algunos aspectos que influyen en los hombres, para esto es necesario hacer el análisis de genero para identificar sobre todo los riesgos a los que se exponen dentro de la cárcel. En el contexto de estudio encontramos a mujeres cumpliendo condenas por diferentes delitos como crímenes, robos u otros factores propios a la conducta humana de la persona que cumple la condena. (Boldo, 2014)

\section{Materiales y métodos}

Durante la investigación participaron 33 mujeres del Centro de Rehabilitación Social Femenino Portoviejo Tomas Larrea, las cuales han sido seleccionadas durante la investigación, se utilizó un diseño instrumental (entrevistas y encuestas). Este trabajo es de enfoque cuantitativo, a través de métodos, técnicas de investigación se recolectaron y analizaron datos que permitieron describir y explicar las variables sobre las condiciones psico sociales de las mujeres privadas de libertad.

Se utilizó el método descriptivo se ocupa de la descripción de datos y características de la población objeto de estudio, se procedió a establecer contacto con los directivos de la institución, 
explicar el motivo de la investigación y posterior a ello se seleccionó a las mujeres privadas de libertad que deseaban colaborar con la investigación.

El método estadístico permitió la secuencia de procedimientos para el manejo cualitativo y cuantitativo de la investigación, tiene como propósito la comprobación del estudio realizado, sobre todo de una parte de la realidad, se basa en recolección, recuento, presentación, síntesis y análisis.

La entrevista, fue realizada con el fin de obtener información sobre las condiciones psicosociales de las mujeres privadas de libertad, aplicados a profesionales y conocedores del tema para tener una perspectiva general de la problemática.

La encuesta, sirvió como estrategia para tomar declaraciones estructurales a las mujeres privadas de libertad a través de un formulario previamente diseñado con preguntas abiertas, cerradas, dicotómicas, jerarquizadas como base de información en el trabajo de investigación.

Una vez aplicados los instrumentos se procedió a analizar las respuestas y se tabularon los resultados, para posterior a ello realizar el análisis e interpretación de la información obtenida de la investigación sobre las condiciones psico sociales de las mujeres privadas de la libertad, datos que estarán reflejados en el trabajo desarrollado.

\section{Resultados.}

Para la presentación de los resultados se hace énfasis a los objetivos planteados en la introducción donde se determina lo siguiente: 


\section{Análisis e interpretación de los resultados}

Resultados de las encuestas aplicadas a las mujeres privadas de libertad del Centro de Rehabilitación Social Femenino Portoviejo "Tomás Larrea, determinando los datos que más prevalecen.

$\begin{array}{ccc}\text { Alternativa } & \text { Frecuencia } & \mathbf{\%} \\ \mathbf{1 8} \text { a 25 } & 14 & 42,42 \\ \mathbf{2 6} \text { a 35 } & 9 & 27,27 \\ \mathbf{3 6} \text { a 45 } & 8 & 24,24 \\ \mathbf{4 6} \text { a 55 } & 1 & 3,03 \\ \mathbf{5 6} \text { a 65 } & 1 & 3,03 \\ \text { Total } & \mathbf{3 3} & 100,00\end{array}$

\section{Tabla 1: Edad de las Mujeres Privadas de Libertad}

Análisis: Según cuadro y grafico estadísticos \# 1 de las mujeres privadas de libertad encuestadas: indican que el $43 \%$ tienen edad entre 18 a 25 años; el 27\% entre 26 a 35 años; el $24 \%$ desde 36 a 46 años; el 3\% entre 46 a 65 años.

Interpretación: Existe un alto porcentaje de mujeres jóvenes recluidas, generando un impacto negativo en el desarrollo personal, económico y social.

\begin{tabular}{|l|r|r|}
\hline Alternativa & Frecuencia & \% \\
\hline Drogas & 9 & 27,27 \\
\hline Asesinato & 8 & 24,24 \\
\hline Robo & 10 & 30,30 \\
\hline Cómplice de violación & 1 & 3,03 \\
\hline Lavado de activo & 1 & 3,03 \\
\hline Asociación ilícita & 4 & 12,12 \\
\hline Total & $\mathbf{3 3}$ & $\mathbf{1 0 0 , 0 0}$ \\
\hline
\end{tabular}

Tabla 2: Delitos cometidos por las Mujeres Privadas de Libertad 
Análisis: Según cuadro y grafico estadístico \# 2, de las mujeres privadas de libertad encuestadas: $31 \%$ ha cometido delitos de robo o hurto; el $27 \%$ por drogas; el $24 \%$ asesinato; $12 \%$ por asociación ilícitas y el 3\% por cómplice de violación y lavado de activos.

Interpretación: La mayoría de mujeres han sido privadas de libertad por ser cómplices de robo o hurto generados particularmente por hombres; sin embrago otros de los mayores delitos es: traficar, exportar, vender o ser mulas de drogas; el asesinar o matar en una mujer no es común en ellas, son sentenciadas por cómplices o planificadoras del asesinato.

\begin{tabular}{|l|r|r|}
\hline Alternativa & Frecuencia & \% \\
\hline Hijos & 19 & 37,25 \\
\hline Madre & 14 & 27,45 \\
\hline Padre & 2 & 3,92 \\
\hline Hermano/as & 2 & 3,92 \\
\hline Esposo/conviviente & 4 & 7,84 \\
\hline Nadie & 5 & 9,80 \\
\hline Otros & 5 & 9,80 \\
\hline Total & $\mathbf{5 1}$ & $\mathbf{1 0 0 , 0 0}$ \\
\hline
\end{tabular}

Tabla 3: Anterior dependencia económica de la Mujer Privada de Libertad

Análisis: El cuadro y grafico estadístico \# 3, indica que el 37\% de los hijos dependían económicamente del trabajo de la mujer antes de estar privada de libertad; el 27\% madre; 10\% otras personas; $8 \%$ esposo o convivientes y el $4 \%$ el padre y los hermanos.

Interpretación: La mujer a pesar de haber delinquido, siempre se ha preocupado por los hijos, siendo fuente económica para los mismos, algunas son madres únicas cuidadoras de los hijos, madre y otros familiares, proveniente de hogares cuya economía es precaria, lo que hace que en muchas ocasiones se explique los delitos cometidos para fines de ayuda económica a familiares. 
Realidades psico-sociales de las mujeres privadas de la libertad en el centro de rehabilitación social femenino Portoviejo "Tomás Larrea"

\begin{tabular}{|l|r|c|}
\hline Alternativa & Frecuencia & \% \\
\hline Excelente & 0 & - \\
\hline Bueno & 2 & 6,06 \\
\hline Regular & 15 & 45,45 \\
\hline Malo & 16 & 48,48 \\
\hline Total & $\mathbf{3 3}$ & $\mathbf{1 0 0 , 0 0}$ \\
\hline
\end{tabular}

Tabla 4: ¿Cómo califica la atención médica dentro del Centro de Rehabilitación Social Femenino Portoviejo Tomás Larrea?

Análisis: El cuadro y grafico estadístico \# 4, de las mujeres privadas de libertad encuestadas: el $49 \%$ califica como mala, la atención recibida dentro del centro de privación; el $49 \%$ regular; $6 \%$ Bueno y el 0\% excelente.

Interpretación: La condición de salud recibida no es adecuada para ellas, la misma condición de ser mujer demanda de médicos especializados, como ginecólogas, dentistas, obstetras, que no solo les trate como un problema común, si no que indague y se preocupen por las molestias que tienen, algunas manifiestan que cuando han sentido dolencias incontrolables acuden al médico del centro de detención teniendo recetas frecuentemente paracetamol o suero oral.

\begin{tabular}{|l|r|r|}
\hline \multicolumn{1}{|c|}{ Alternativa } & Frecuencia & \multicolumn{1}{c|}{ \% } \\
\hline Depresión & 23 & 21,90 \\
\hline Soledad & 18 & 17,14 \\
\hline Violencia & 2 & 1,90 \\
\hline Adicción a sustancias prohibidas & 6 & 5,71 \\
\hline Separación de vínculos familiares & 18 & 17,14 \\
\hline Cambios físicos & 11 & 10,48 \\
\hline Desvalorización personal & 12 & 11,43 \\
\hline Manipulación & 1 & 0,95 \\
\hline Dependencia & 3 & 2,86 \\
\hline Sentimientos suicidas & 9 & 8,57 \\
\hline Otros & 2 & 1,90 \\
\hline Total & $\mathbf{1 0 5}$ & $\mathbf{1 0 0 , 0 0}$ \\
\hline
\end{tabular}

Tabla 5: Consecuencias generadas por la Privación de Libertad 
Análisis: De acuerdo con el cuadro y grafico estadístico \# 5, de las mujeres privadas de libertad encuestadas: el 22\% respondieron que la privación de libertad ha causado en ellas depresión; el 17\% separación de vínculos familiares y soledad; 10\% cambios físicos; $11 \%$ desvalorización de imagen, 9\% sentimientos suicidas; $6 \%$ adicción a sustancias; $3 \%$ dependencia; $2 \%$ violencia y el $1 \%$ manipulación.

Interpretación: Las internas al estar en prisión, empiezan a tener patologías psicológicas como la depresión y soledad generadas por el encierro y la separación de los vínculos familiares, pues el hecho de estar encerrada implica el desbordamiento de su imagen y personalidad, estas consecuencias tienden a renacer una mujer diferente ; en pocas ocasiones el estar encerrada tiene impacto positivo, se dan cuenta de todo lo que nunca valoraron, sin embargo en su mayoría tiene efectos como: la adicción a sustancias ( cigarrillo, alcohol, marihuana, heroína, tranquilizantes, inhalantes), lo que conlleva la destrucción constante del organismo de la mujer, haciéndola susceptibles a enfermedades, relaciones sentimentales y sexuales en prisión.

Otro cambio provocado, es el físico, pues la mujer disminuye su peso, la comida y la rutina diaria hace que la mujer no conciba la existencia, adoptando instinto de violencia, manipulación, dificultad para conciliar el sueño, patologías de percepciones sensoriales (visión, audición, gusto, olfato.), problemas de privación sexual, etc.

A raíz de la depresión, angustia, desvalorización personal, devaluación de imagen, aparecen los sentimientos suicidas, en ocasiones intentos por desaparecer del mundo (cortes en las venas, absorción de veneno, intentos de ahorcamiento y otras formas de atentar contra la propia vida). 


\section{Discusión.}

El trabajo de investigación ha cumplido satisfactoriamente con el objetivo general: investigar las condiciones psico sociales de las mujeres que permanecen recluidas en el Centro de Rehabilitación Social Femenino Portoviejo "Tomas Larrea", enfocado en la experiencia de las mujeres privadas de libertad, que se dan dentro del reclusorio, el estar en una prisión para la mujer, no solo significa cumplir una sentencia, más bien significa una condena por la ruptura parcial o total de sus vínculos familiares, convirtiéndose en un hecho doloroso, brusco, que genera depresión, angustias, sufrimiento constante:

- Ruptura del vínculo conyugal.

- Alejamiento y rechazo de los hijos/as.

- Inestabilidad emocional y desviación de conductas en niños/as de la mujer privada.

- Pérdida de la custodia total o temporal de los hijos/as.

- Resentimiento y humillaciones familiares.

Las condiciones físicas no son adecuadas en todos los centros de rehabilitación social, sobre todos para las mujeres que demandan de más necesidades, por ejemplo, el Centro de Rehabilitación Social Femenino Portoviejo "Tomás Larrea", tiene condiciones e infraestructuras antiguas de aproximadamente 60 años y no es adecuado para el modelo de gestión actual, sin embargo, tienen las mejores condiciones en alimentación, vivienda, salud en las mujeres. (Valverde Valverde, 2015)

Las condiciones legales en la actualidad han cambiado, a favor de los privados de libertad, los juicios legales están más ágiles y rápidos, si no tienen abogados el departamento jurídico del 
centro les ayuda a conseguir uno del Estado para poder actuar en la defensa y seguir los procesos correspondientes.

En cuanto a los objetivos específicos planteados como determinar los imaginarios que circulan sobre los estigmas de la mujer común, "mujer, esposa, madre”, generando en ellas cambios físicos, psicológicos y sociales, realizar un análisis de cómo el aislamiento afecta a las mujeres privadas de libertad y cómo ellas afrontan esta situación, desde el momento en que es arrestada la mujer, empieza a sentir consecuencias psicológicas, la desesperación y la angustia del saber cómo será el lugar a donde será trasladada, el abandono de su familiares, el escándalo de la prensa y comentarios de la sociedad, hacen que vayan optando conductas depresivas, cambios fisiológicos, traumas de encierro por la adaptación del sistema penitenciario, crisis de ansiedad, perturbaciones en el sueño, inestabilidad emocional y sexual, irritabilidad que lleva a la violencia, consumo de sustancias, desconcentración y distorsión de la realidad. (Constant, 2012)

Cuando alguien comete un delito, es acusado y señalado por la sociedad, el hecho de que la mujer este en una cárcel significa que ella ha violentado su rol y han quebrantado leyes, por tal es calificada como lo peor mujer, esposa, madre, hija, son discriminadas ya que para ellos son personas que causaron o causarán daño estando fuera de la cárcel; luego al querer ser reinsertadas las personas no les ofrecen una nueva oportunidad por los perjuicios morales y sociales que imponen al no conocer la realidad del sistema carcelario. (Tracin Robinson, 2015)

Las mujeres por lo general no cometen delitos que forjen el riesgo o asesinato de otros individuos, normalmente son planificadores, mentalizadoras, expendedoras y vendedoras, unas que otras actoras de crímenes, se reflejan delitos frecuentes entre ellos: 
- $\quad$ Asociación ilícita

- $\quad$ Robo simple, agravado

- $\quad$ Drogas (expedición, mulas, tráficos, tenencia ilegal, ventas)

- Cómplices de asesinatos

- $\quad$ Extorsión

- $\quad$ Cómplices de Delito Sexual

- Trata de blanca

- Estafas

Las mujeres no todas delinquen porque quieren o por placer, algunas lo hacen por necesidad económica y sustentación de la familia; sin embargo, cualquiera que sea la causa, todas tienen algún antecedente de violencia familiar, abuso sexual, padres drogadictos, alcohólicos, familia disfuncional, etc. (Samaranch, 2012)

Hay que destacar que el gobierno actual se está preocupando por el sistema carcelario, invierte en los centros penitenciarios y en la reinserción social de los mismos a través de convenios y actividades, respetando sus derechos y obligaciones en diferentes ámbitos de salud, alimentación, educación y todo lo referente al contorno. (Abelardo Sánchez, 2014)

El Centro de Rehabilitación Social Femenino Portoviejo “Tomás Larrea”, consta de departamento médico, legal, psicológico, trabajo social, laboral, diagnóstico y evaluación, educativo (Profesores, Escuela de Educación Básica, Biblioteca) y mantiene convenios con instituciones como: Ministerio de la Cultura, Deporte, Servicio Ecuatoriano de Capacitación Profesional, Universidad Laica Eloy Alfaro de Manabí, Universidad Técnica de Manabí. Patronato Municipal, Colegios, Ministerio de la cultura, Deporte, Economía, Organismos no gubernamentales, Iglesias y 
otras que ofrecen, cursos de manualidades, área de talleres, danza, teatro, bailo terapia, cultura física, lo que hace que las mujeres ocupen y distraigan la mente dentro de la prisión.

Se le integra a la mujer en actividades sociales, culturales, educativas, religiosas, sin discriminación aportando a su reinserción social, familiar. (León, 2014).

\section{Conclusiones.}

Después de haber realizado el presente trabajo de investigación, se concluye lo siguiente:

Se logró cumplir con los objetivos propuestos porque se conoció las condiciones psico sociales de las mujeres privadas de la libertad del Centro de Rehabilitación Social Femenino Portoviejo, donde se determinaron algunos factores que influyen en la vida carcelaria que las conlleva a tomar diferentes actitudes en el entorno en que se desenvuelven y es aquí donde surge la importancia del apoyo psicológico a través de profesionales especialistas.

Hubo apertura por parte de las autoridades del Centro de Rehabilitación para desarrollar la investigación, lo que permitió culminar el estudio para la obtención de resultados que se reflejan sobre todo en el análisis de las encuestas realizadas.

Las mujeres que están en el centro penitenciario Tomás Larrea tienen antecedentes de haber sido víctimas de violencia familiar e intrafamiliar, abusos sexuales. Las condiciones psicológicas manifestadas son: temor, angustia, depresión, soledad y sentimientos suicidas, lo que las hace vulnerables al consumo de sustancias, conductas violencias, desviación de roles sexuales e identidad, desvalorización personal, reproche constante de lo cometido y de la pérdida parcial de su libertad, lazos familiares, condiciones sociales, económicas, laborales; esto se muestra en la 
convivencia carcelaria donde aluden pérdidas profundas, dolorosas, rupturas y mortificaciones externas, creándose diariamente una nueva identidad en la mujer.

Las condiciones que la sociedad impone sobre las mujeres privadas de libertad se enmarcan en el rol que ejercen, el hecho de ser madre, esposa, hija hace que la mujer se vea como un ser perverso, malo que ha irrumpido las reglas sociales cuando es encarcelada.

Las reclusas del Centro de Rehabilitación Social Femenino de Portoviejo Tomás Larrea tienen condiciones económicas medias, los familiares les ayudan para gasto de sustentación dentro de la prisión; sin embargo, a pesar de su condición aportan económicamente al hogar a través de la realización y venta de manualidades, gastronomía, lavado de ropa, que realizan dentro de prisión.

Después de ingresar a prisión las condiciones familiares se deterioran, algunas mantienen los lazos familiares y son visitadas frecuentemente por los hijos y familiares, los niños/as reciben un fuerte impacto, en la usencia de la figura materna, tienden a la depresión, bajo rendimiento escolar, aislamiento e inestabilidad emocional.

Las condiciones de salud en las internas son buenas, la mayoría no tienen ninguna enfermedad, sin embargo, la atención médica del Centro de Rehabilitación Social Femenino de Portoviejo Tomás Larrea no es la adecuada, ya que no cuentan con especialistas que atiendan las demandas de salud.

\section{Bibliografía.}

Abelardo Sánchez, L. (2014). Economía del espacio carcelario amplio. Lima: Bulletin.

Boldo, C. S. (2014). Las cárceles de mujeres en México. Revista de las Ciencias Sociales de la Universidad Iberoamericana, 9(17), 1-27. 
Constant, C. (2012). Relaciones entre internas y guardias en el penal de mujeres de Lima. Lima: Bulletin.

Estibaliz de Miguel, C. (2014). Encarcelamiento de mujeres. El castigo penintenciario de la exclusión social y desigualdad de género. ZERBITZUAN, 56(1), 75-86.

León, A. S. (2014). Pensar las cárceles de América Latina. Lima: Bulletin.

Mapelli, C., Borja Herrera, M., \& Sordi Stock, M. (2013). La exclusión de las excluidas. Sevilla: Artículo Derecho Penal y Ciencias Criminales, Universidad de Sevilla.

Rodriguez, E. B. (2015). Mujeres y cárceles en América Latina. Barcelona- España: Redi.

Samaranch, E. (2012). Mujeres, cárceles y drogas. España: Board.

Tracin Robinson. (2015). Casos de mujeres privadas de la libertad por emergencia obstétrica durante el embarazo. Salvador: Human Rights.

Valverde Valverde, Y. (2015). Efecto de un programa de actividades físicas sobre la calidad de vida en las mujeres privadas de la libertad. Costa Rica: Repositorio académico institucional de la Universidad Nacional de Costa Rica. 